Document downloaded from:

http://hdl.handle.net/10251/57567

This paper must be cited as:

Doménech Gómez, JD.; Sanchez Fandiño, JA.; Gargallo Jaquotot, BA.; Muñoz Muñoz, P. (2014). Arbitrary Coupling Ratio Multimode Interference Couplers in Silicon-on-Insulator. Journal of Lightwave Technology. 32(14):2536-2543. doi:10.1109/JLT.2014.2329994.

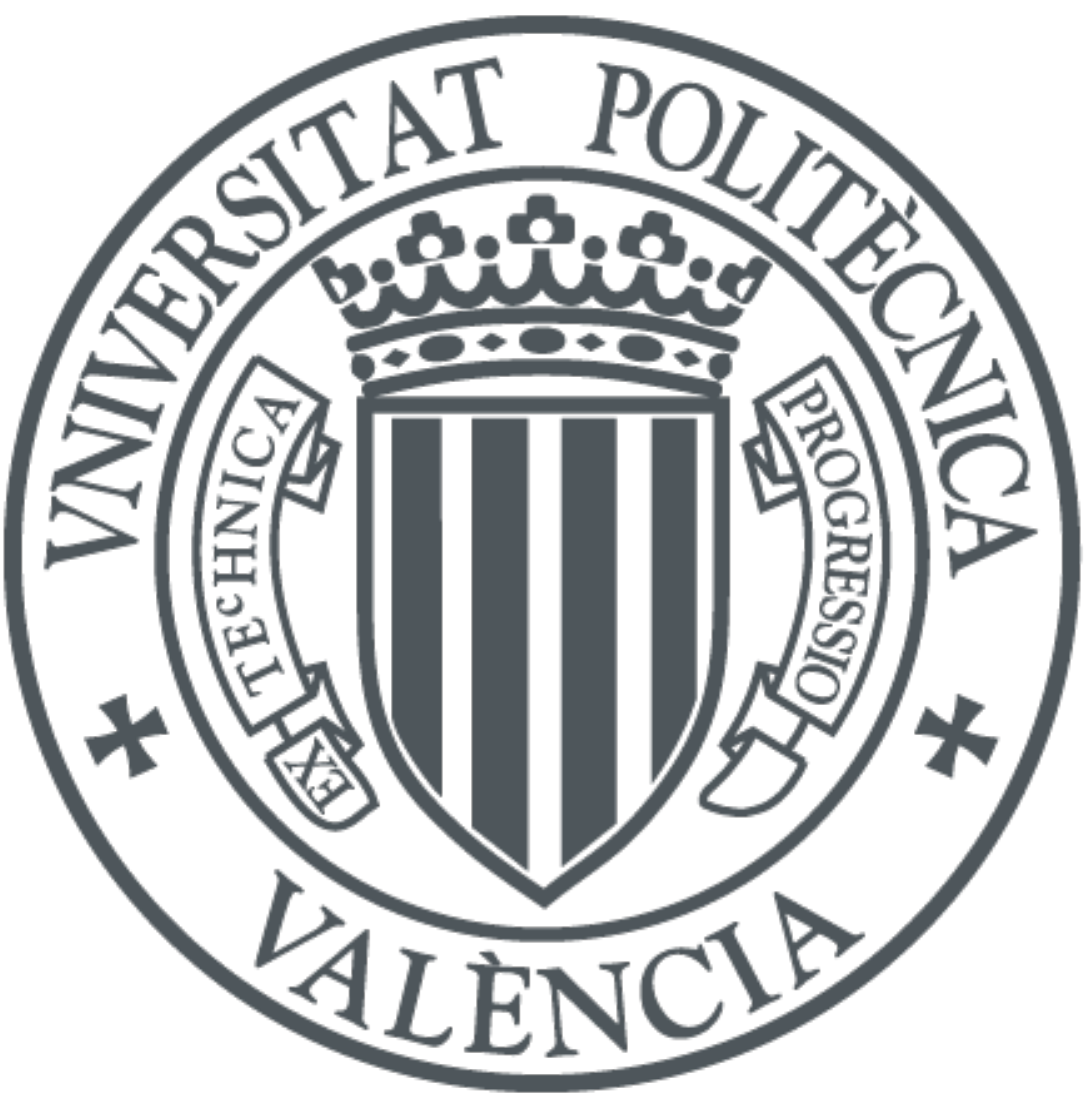

The final publication is available at

http://dx.doi.org/ 10.1109/JLT.2014.2329994

Copyright Institute of Electrical and Electronics Engineers (IEEE)

Additional Information 


\title{
Arbitrary coupling ratio multimode interference couplers in Silicon-on-Insulator
}

\author{
José David Doménech, Javier S. Fandiño, Bernardo Gargallo and \\ Pascual Muñoz Senior Member, IEEE, Member, OSA
}

\begin{abstract}
In this paper we present the design, manufacturing, characterization and analysis of the coupling ratio spectral response for Multimode Interference (MMI) couplers in Siliconon-Insulator (SOI) technology. The couplers were designed using a $\mathrm{Si}$ rib waveguide with $\mathrm{SiO}_{2}$ cladding, on a regular $220 \mathrm{~nm}$ film and $2 \mu \mathrm{m}$ buried oxide SOI wafer. A set of eight different designs, three canonical and five using a widened/narrowed coupler body, have been subject of study, with coupling ratios 50:50, 85:15 and 72:28 for the former, and 95:05, 85:15, 75:25, 65:35 and 55:45 for the latter. Two wafers of devices were fabricated, using two different etch depths for the rib waveguides. A set of six dies, three per wafer, whose line metrology matched the design, were retained for characterization. The coupling ratios obtained in the experimental results match, with little deviations, the design targets for a wavelength range between 1525 and $1575 \mathrm{~nm}$, as inferred from spectral measurements and statistical analyses. Excess loss for all the devices is conservatively estimated to be approximately $0.6 \mathrm{~dB}$ in average. All the design parameters, body width and length, input/output positions and widths, and tapers dimensions are disclosed for reference.
\end{abstract}

Index Terms-Multimode interference coupler, Silicon-onInsulator, coupling ratio, variation analysis, performance statistics.

\section{INTRODUCTION}

Optical couplers are perhaps one of the most basic and most used among the building blocks for photonic integrated circuits (PICs) in all currently available technology platforms [1]. Different integrated implementations exist (see [2]), and they are usually compared according to their coupling constant and operational wavelength range. Among all of them, the Multimode Interference (MMI) coupler is mostly used in high index contrast PIC technologies, such as III-V and group IV materials, since it is in general more compact and preserves the coupling constant over a wide wavelength range. Since its inception by Ulrich in 1975 [3], and the demonstrations of MMIs as we know them today carried out by Pennings and co-workers [4], multitude of papers have studied the different aspects of these very versatile devices: fundamental theory and design rules for the so called canonical MMIs, by Soldano [5] and Bachmann [6], [7]; design rules and experimental demonstrations of widened/narrowed body MMIs for arbitrary coupling ratios at a single wavelength by Besse [8], with reconfiguration using thermal tuning by Leuthold [9]; tolerance

J.D. Doménech is with VLC Photonics S.L., C/ Camino de Vera s/n, Valencia 46022, Spain e-mail: david.domenech@vlcphotonics.com

B. Gargallo, J.S. Fandiño and P. Muñoz are with the Optical and Quantum Communications Group, iTEAM Research Institute, Universitat Politècnica de València, C/ Camino de Vera s/n, Valencia 46022, Spain email: pmunoz@iteam.upv.es.

Corresponding author: P. Muñoz, e-mail: pmunoz@iteam.upv.es. analysis by Besse [10]; design optimization for different technologies by Halir [11]; library of experimentally demonstrated 50:50 Silicon-on-Insulator couplers [12], to name a few. There are other means of implementing couplers with arbitrary ratio that make use of additional structures, as for instance the combination of two MMIs in a Mach-Zehnder Interferometer (MZI) like structure recently proposed by Cherchi [13].

In this paper we report on the design and experimental demonstration of arbitrary coupling ratio MMIs following the design rules by Besse and co-workers [8], supported by Beam Propagation Method (BPM) commercial software optimization [14], on a Silicon-on-Insulator (SOI) platform. Complimentary to previous works available in the literature this paper presents:, a) all the design parameters required to obtain broadband (1525-1575 nm) coupling ratio, with modest excess loss, for canonical 50:50, 85:15 and 72:28 MMIs, as well as for widened/narrowed 95:05, 85:15, 75:25, 65:35 and 55:45 MMIs are disclosed; b) spectral traces demonstrating the otherwise well known theoretically broadband operation of these devices; c) statistics for the coupling ratio variations in the operational wavelength range, that may be of use to perform variation analysis of more complex on-chip devices, circuits and networks based on these MMIs; d) explanation on how measurement deviations, due to variations in the in/out coupling to/from the chip, can bias the coupling ratio results, and e) measurements to infer the reproducibility, die to die and wafer to wafer, of the responses. These reference designs, experimentally validated, together with the statistical variations and reproducibility information, can be used as starting point for other designers and researchers of these devices, and of more complex chip networks employing them, on SOI platforms.

\section{DESIGN}

The design of all the MMIs was carried out in three steps: i) cross-section analysis and 2D reduction, ii) analytic approach and iii) numerical BPM optimization. The cross section consists of a buried oxide layer of 2 microns height, capped with a $220 \mathrm{~nm} \mathrm{Si}$ layer and a $\mathrm{SiO}_{2}$ over-cladding. Rib waveguides, with $130 \mathrm{~nm}$ etch depth from top of the $\mathrm{Si}$ layer, were used in the design stage. For the same lithographic resolution, rib waveguides provide more robust MMIs than strip waveguides, owing to the fact that wider waveguides are required to support the same number of modes [5]. This comes at the cost of increased footprint and some additional design refinements are required to minimize the MMI imbalance and 
excess loss [11] [15], besides the complexity of two mask level fabrication described in [16]. The latter trade-off is common in applications where the coupling constant needs to be set very precisely, for instance in very small free spectral range Mach-Zehnder interferometers (MZI), to compensate for the significantly larger loss difference between the long and short interferometer arms [17]. Moreover it is determinant for onchip reflectors based on Sagnac interferometers, where the reflectivity is solely determined by the coupling ratio of the coupler in the interferometer [18].

Firstly, for the cross-section analysis a film-mode matching mode solver was used [14]. The wavelength dependence of the refractive indices was included in the solver (see the Appendix). For a given MMI width, the first and second mode propagation constants, $\beta_{0}$ and $\beta_{1}$ respectively, were found for a wavelength of $1.55 \mu \mathrm{m}$ for TE polarization, and the beat length $L_{\pi}=\pi /\left(\beta_{0}-\beta_{1}\right)$ was computed from these. For the case of all the MMIs subject to design, the body width was set to $10 \mu \mathrm{m}$. The effective indices for the first and second mode given by the solver are $\mathrm{n}_{e f f, 0}=2.84849$ and $\mathrm{n}_{e f f, 1}=2.84548$. Therefore the beat length results into $\mathrm{L}_{\pi}=257.61 \mu \mathrm{m}$. In order to later use a 2D BPM method, the cross-section was reduced vertically to a $1 \mathrm{D}$ waveguide using the effective index method (EIM) [19]. EIM was firstly used to derive the 1D effective index for the core region, and then the effective index left/right to the core was calculated by numerically solving (with a bisection method) for the 1D modes of the reduced structure to match the previously calculated $L_{\pi}$ on the 2D cross-section.

Secondly, analytic design rules for canonical [5] and arbitrary coupling ratio [8] MMIs were used. These rules provide, for a given MMI width, an analytic approximation for the MMI body length, named $\mathrm{L}^{0}$, from the previously calculated $L_{\pi}$, and for the case of arbitrary ratio, the width variation and body geometry (named type A, B, B symmetrized, C and D in [8]). For completeness, the analytic approximations for the MMI lengths are reproduced here:

$$
\begin{aligned}
L_{A}^{0} & =\delta_{W}^{A} \frac{1}{2}\left(3 L_{\pi}\right) \\
L_{B, B s y m}^{0} & =\delta_{W} \frac{1}{3}\left(3 L_{\pi}\right) \\
L_{C}^{0} & =\delta_{W} \frac{1}{4}\left(3 L_{\pi}\right) \\
L_{D}^{0} & =\delta_{W} \frac{1}{5}\left(3 L_{\pi}\right)
\end{aligned}
$$

where $3 L_{\pi}$ is the distance for the first direct (not mirrored) image [5], $\delta_{W}^{A}=1-\Delta W / W$ and $\delta_{W}=1-2 \Delta W / W$, with $W$ the MMI body width and $\Delta W$ the MMI body widening/narrowing. Note in the case of rectangular body, $\Delta W=0$ and the last two expressions are equal to 1 . Up to this stage, only the MMI body width and a first guess for the length are set.

The final step consists of using BPM for a MMI having input/output tapered waveguides. Tapers are required to minimize the MMI excess loss, imbalance and reflections as described in [11] [15]. Hence, BPM is used to find iteratively both the MMI length and the input/output tapers width. The optimization process has as target to minimize the coupler imbalance, i.e. that the ratios at both outputs match the target, and to maximize the overall optical power with respect to the input, i.e. to minimize the excess loss. To do so, the BPM is equipped with mode overlap monitors at the output waveguides. They provide the amplitude and phase for the overlap of the output field with the fundamental mode of the waveguide. The optimization process starts with a fixed set of taper width and MMI length. The starting taper width was set to $3 \mu \mathrm{m}$. The taper length was set to $50.0 \mu \mathrm{m}$, which is sufficiently large for adiabatic linear tapering as per [20]. The MMI length was set to the values obtained through the aforementioned analytic formulas. They provide an MMI length that does not account the tapering of the input/outputs, which in turn modifies the propagation conditions in the multimode waveguide. Therefore, for the initial guess of taper width, the length of the MMI is solved numerically in a first step. Next, the width of the taper is varied. Both parameters are iteratively changed following update and minimization numerical methods commonly now, until a solution is found for the coupling ratios, having as stop condition a tolerance of 0.01 . The optimization was performed firstly for $\lambda=1.55 \mu \mathrm{m}$, and finally cross-checked for the design wavelength interval, $1.525-1.575 \mu \mathrm{m}$. The body shapes and parameters for the MMIs are given in Fig. 1 and Table I. The parameters subject to numerical optimization are marked in the table with the * symbol.

Note the optimization process yields shorter MMI body lengths than those provided by the analytic expressions in Eqs. (1)-(4). This can be explained in terms of the underlying physics as follows. The analytic expressions provide the length for a perfectly rectangular body. Including input/output tapers perturbs the rectangular body shape in the longitudinal dimension, producing multimode propagation in a length larger than the canonical rectangular length. The effect is similar to having a perfectly rectangular body, but with increased length. Hence, to compensate this extra propagation length in the tapers, the body length needs to be reduced.

\section{EXPERIMENTAL RESULTS}

\section{A. Fabrication}

The designs were fabricated on two different wafers, named A and B from now onwards, using a $248 \mathrm{~nm}$ CMOS line MultiProject Wafer at the Institute of Micro-Electronics, Singapore. The difference between wafers A and B was the etch depth for the rib waveguides, $130 \mathrm{~nm}$ (as per design) and $160 \mathrm{~nm}$ from top of the Si film, respectively. From the dies delivered by the fab, those exhibiting metrology (grating line width, grating space width, waveguide width) close to target were retained for measurements. The target grating line and space were both $315 \mathrm{~nm}$. Metrology shows grating line in [321,333] nm, and grating space in [296-312] $\mathrm{nm}$. The target waveguide width for the process calibrations at the fab was $500 \mathrm{~nm}$, and metrology shows widths in $[520,541] \mathrm{nm}$. The number of dies with metrology in these ranges amounted for 3 dies per wafer, namely A1, A2 and A3 for wafer A, and B1, B2, B3 for wafer B. A picture of the fabricated devices is shown in Fig. 2. All the layouts included focusing grating couplers (FGC) to couple 


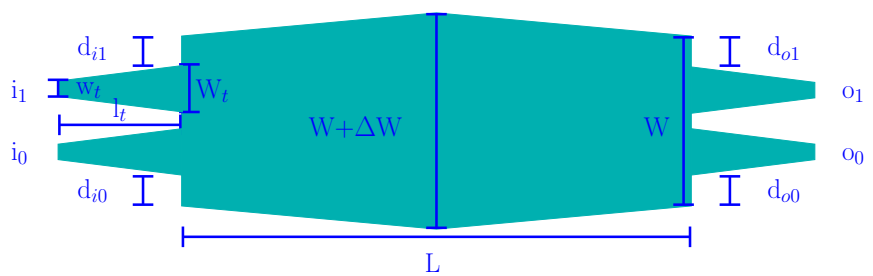

(a)

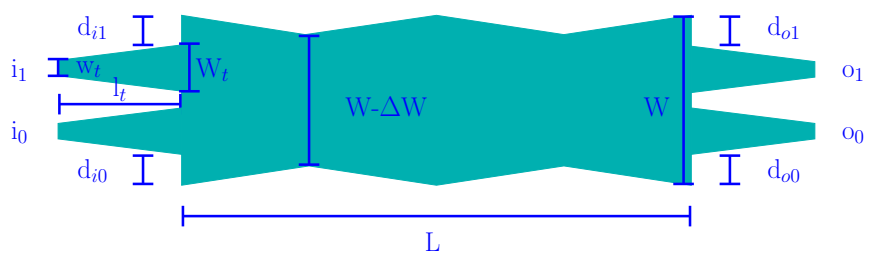

(b)

Fig. 1. Multimode Interference coupler geometries employed in this work, (a) Type A, C, D layouts and (b) Type B Symmetrized layout. Abbreviations: $L$ and $W$, MMI body length and width respectively; $d_{i o}$ distance of input/output waveguides from the edges of the MMI body; $l_{t}$ input/output waveguide taper length; $w_{t}$ and $W_{t}$ input/output taper narrow and wide side widths, respectively.

\begin{tabular}{|c|c|c|c|c|}
\hline \multicolumn{5}{|c|}{ MMI Design Parameters } \\
\hline Id & $\# 1$ & $\# 2$ & $\# 3$ & $\# 4$ \\
\hline \hline Ratio & $50: 50$ & $85: 15$ & $95: 05$ & $85: 15$ \\
\hline Type & $\mathrm{A}$ & $\mathrm{C}$ & $\mathrm{B} \mathrm{Sym}$ & $\mathrm{B} \mathrm{Sym}$ \\
\hline $\mathrm{L}^{0}$ & $\delta_{W}^{A}(1 / 2) \mathrm{L}_{\pi}$ & $\delta_{W}(3 / 4) \mathrm{L}_{\pi}$ & $\delta_{W}(3 / 3) \mathrm{L}_{\pi}$ & $\delta_{W}(3 / 3) \mathrm{L}_{\pi}$ \\
& 128.81 & 193.21 & 220.40 & 192.75 \\
\hline $\mathrm{L}^{*}$ & 122.96 & 184.95 & 211.95 & 184.55 \\
\hline $\mathrm{W}$ & 10.00 & 10.00 & 10.00 & 10.00 \\
\hline$\Delta \mathrm{W}$ & 0 & 0 & 0.72 & 1.26 \\
\hline $\mathrm{d}_{i 0}, \mathrm{~d}_{i 1}$ & 1.90 & 0.83 & 1.97 & 1.97 \\
\hline $\mathrm{d}_{o 0}, \mathrm{~d}_{o 1}$ & 1.90 & 0.83 & 1.97 & 1.97 \\
\hline $\mathrm{l}_{t}$ & 50.00 & 50.00 & 50.00 & 50.00 \\
\hline $\mathrm{W}_{t}$ & 0.45 & 0.45 & 0.45 & 0.45 \\
\hline $\mathrm{W}_{t}^{0}$ & 3.00 & 3.00 & 3.00 & 3.00 \\
\hline $\mathrm{W}_{t}{ }^{*}$ & 2.75 & 3.35 & 2.7 & 2.7 \\
\hline $\mathrm{Body}$ & Single & Single & Double & Double \\
\hline \hline $\mathrm{Id}$ & $\# 5$ & $\# 6$ & $\# 7$ & $\# 8$ \\
\hline \hline $\mathrm{Ratio}$ & $75: 25$ & $65: 35$ & $55: 45$ & $72: 28$ \\
\hline $\mathrm{Type}$ & $\mathrm{C}$ & $\mathrm{D}$ & $\mathrm{D}$ & $\mathrm{D}$ \\
\hline $\mathrm{L}^{0}$ & $\delta_{W}(3 / 4) \mathrm{L}_{\pi}$ & $\delta_{W}(3 / 5) \mathrm{L}_{\pi}$ & $\delta_{W}(3 / 5) \mathrm{L}_{\pi}$ & $\delta_{W}(3 / 5) \mathrm{L}_{\pi}$ \\
& 257.49 & 177.66 & 208.55 & 154.57 \\
\hline $\mathrm{L}^{*}$ & 247.18 & 170.36 & 200.04 & 147.76 \\
\hline $\mathrm{W}$ & 10.00 & 10.00 & 10.00 & 10.00 \\
\hline$\Delta \mathrm{W}$ & 3.26 & 1.5 & 3.34 & 0 \\
\hline $\mathrm{d}_{i 0}, \mathrm{~d}_{i 1}$ & 1.11 & $0.60,2.60$ & $0.61,2.61$ & $0.75,2.75$ \\
\hline $\mathrm{d}_{o 0}, \mathrm{~d}_{o 1}$ & 1.11 & $2.60,0.60$ & $2.61,0.61$ & $2.75,0.75$ \\
\hline $\mathrm{l}_{t}$ & 50.00 & 50.00 & 50.00 & 50.00 \\
\hline $\mathrm{w}_{t}$ & 0.45 & 0.45 & 0.45 & 0.45 \\
\hline $\mathrm{W}_{t}^{0}$ & 3.00 & 3.00 & 3.00 & 3.00 \\
\hline $\mathrm{W}_{t}{ }^{*}$ & 2.83 & 2.83 & 2.80 & 2.50 \\
\hline $\mathrm{Body}$ & Single & Single & Single & Single \\
\hline
\end{tabular}

TABLE I

MMI DESIGN PARAMETERS, LENGTHS AND WIDTHS IN $\mu m$. PARAMETERS MARKED WITH THE SYMBOL $*$ WERE SUBJECT TO NUMERICAL OPTIMIZATION. $\delta_{W}=1-2 \Delta W / W . \delta_{W}^{A}=1-\Delta W / W$

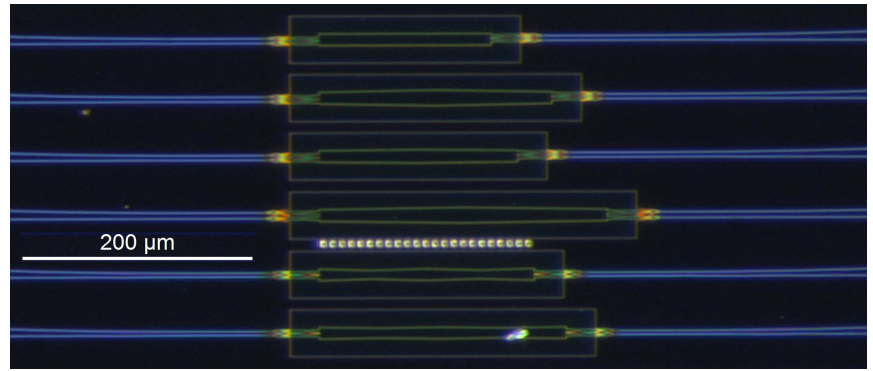

Fig. 2. Chip photograph for the fabricated MMI devices, from bottom to top MMIs \#3-\#8, i.e. 95:05, 85:15, 75:25, 65:35, 55:45 (non canonical) and 72:28 (canonical) splitting ratios.

light vertically into/out from the chips [21]. Both the FGCs and the waveguides connected to the MMIs supported only TE polarization.

\section{B. Characterization setup}

The characterization setup consists of a set of three motorized positioners. Two of them are used for holding the fibers vertically at the right angle to couple light into the FGCs $\left(10^{\circ}\right.$ from the normal to the chip surface), whereas the third one holds the sample on top of a thermally controlled $\left(25^{\circ} \mathrm{C}\right)$ vacuum chuck. A CCD camera vision system is also mounted in a motorized stage and a LED lamp is used for illumination. For the measurements, the fibers are aligned manually in two steps. Firstly, the fibers are approximated to the FGC locations by visual inspection using the live images from the camera. The approximated height location can be obtained when the fiber and its shadow overlap. Secondly, a broadband light source is connected to one of the fibers, whereas a power meter is connected at the end of the other. Hence, the positions of the input and output fibers are optimized with the motorized stages to obtain the maximum power. A further detailed description of this setup and procedure can be found in [22]. After the fibers alignments are optimized, an Optical Spectrum Analyzer (OSA) is used to record the spectra with a resolution of $10 \mathrm{pm}$.

\section{Test structures and stability}

Prior to measuring and processing the target devices, straight waveguides were measured in order to gather information on the different features observed in preliminarily recorded traces. Referring to Fig. 3, a single straight waveguide was measured repeatedly, and a set of 8 consecutive traces was obtained. These are shown in black dots in the figure, with the average in marine blue solid line. Some Fabry-Pérot (FP) like ripples were observed, with a separation between peaks of $0.26 \mathrm{~nm}$. These are attributed to reflections occurring elsewhere, and that are otherwise not present in the spectrum of the optical source.Thus a moving average of 71 points (traces were recorded with a spectral resolution of $10 \mathrm{pm}$, this corresponds to $0.71 \mathrm{~nm}$, approximately twice $\Delta \lambda$ ) was applied to the 8 traces, in order to clean the FP peaks. The results are shown in Fig. 3 with red dots for the 8 traces, whereas the average for them is shown in a dashed green line. 


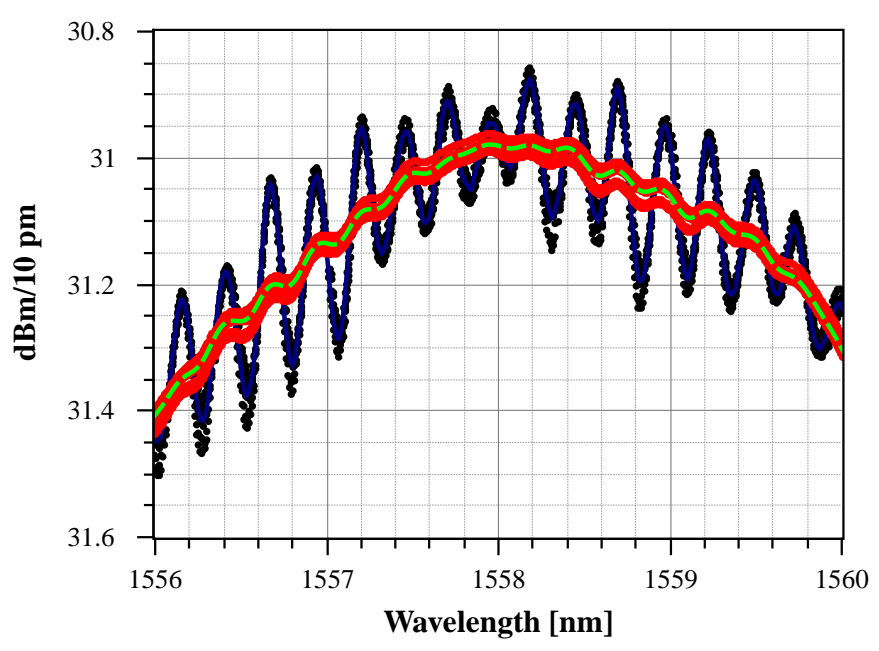

Fig. 3. Spectral traces recorded consecutively for one straight waveguide. The black dots correspond to 8 traces, while their average is shown in marine blue solid line. The red dots correspond to the former 8 traces smoothed with a moving average of 71 points, and the green dashed line to its average.

Hence, all spectral traces recorded for the MMIs are to be smoothed as described, before using them in the calculations of coupling ratios described in the next subsection. As a final remark on stability, each trace involved a sweep in the OSA of 20 seconds. From Fig. 3 a good setup stability can be inferred, i.e. very little power variations due to mechanical issues, as the fiber holders, translation stages, tabletop and other during the time it took to acquire these 8 traces (close to 3 minutes) is attained. Though not shown, minor drifts in the alignments started to happen right after the time for the 8 traces. For the MMIs a single trace is collected per output, therefore, it is not subject to the mechanical drifts of the measurement setup.

\section{MMIs Coupling ratio}

The coupling ratios for the MMIs were derived from the spectral traces measured at outputs 'm', from input ' 1 ', termed $S_{l, m}(\lambda)$, as:

$$
K_{l, m}(\lambda)=\frac{S_{l, m}(\lambda)}{S_{l, 0}(\lambda)+S_{l, 1}(\lambda)}
$$

where $l=0,1$ and and $m=0,1$ label the input and output waveguides respectively, and with spectral traces in linear units. $K_{l, m}(\lambda)$ traces for MMI\#2 and MMI\#3 on die A1 are plotted in Fig. 4. The results show good agreement with target coupling ratios, where deviations are approximately in the range of \pm 0.01 . For the rest of the dies, similar spectral traces and deviations were obtained from both wafers, albeit the etch depth difference between wafer A and B. Note the additional etch depth of $30 \mathrm{~nm}$ in wafer B did not change significantly the results, which is in good agreement with the sensitivity analysis reported in [11]. The results for all the couplers per die and wafer are compiled in summary graphs given in Fig. 6 and Fig. 7, where the average coupling ratios and standard deviations in the wavelength range of the measurements are shown.

MMI \#1 50:50 samples exhibited coupling constants around 0.5 with deviations in the whole wavelength range of less than

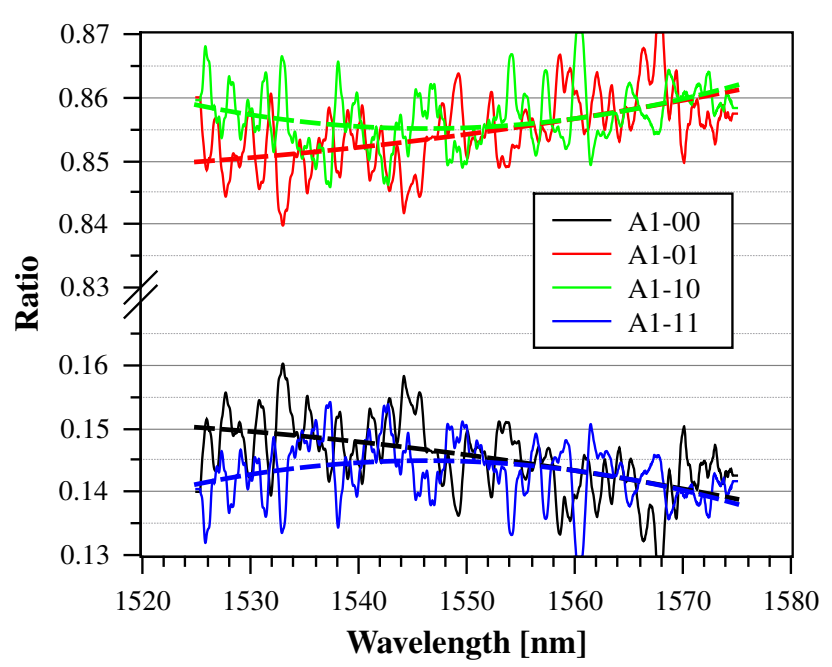

(a) MMI\#2

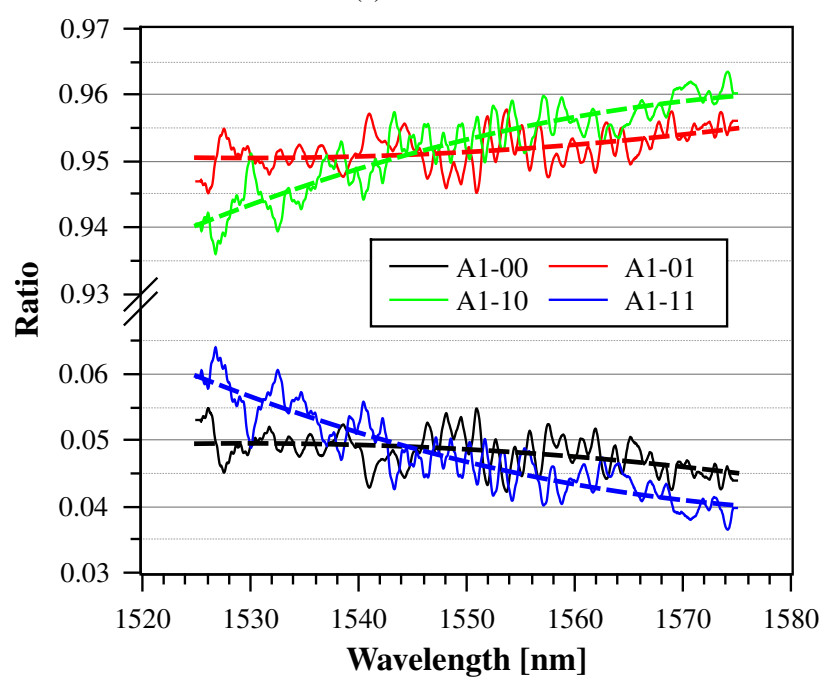

(b) MMI\#3

Fig. 4. Spectral coupling ratio for canonical 85:15 MMI (a) and narrowed 95:05 MMI (b) in solid lines, with second order polynomial fit in dashed thick lines.

\pm 0.01 for all dies, except A1 and B3, where some imbalance is clearly appreciated. For MMIs \#2 to \#4 the graphs are given with broken axes, but with the same interval around the target coupling ratio. Comparing MMI \#2 and \#4, which have as target 85:15 splitting ratio, the performance of the first proved to be best for all dies. One might be tempted to attribute this to the fact that device \#2 is a canonical (rectangular body) design, whereas \#4 follows the widened body geometry shown in Fig. 1-(b), i.e. Type B Symmetrized. However MMI \#3 shown in Fig. 6-(c) exhibits very good performance, which might be misleadingly interpreted as MMI \#4 being a suboptimal design. Therefore additional insight is provided in the following. Note the spectral traces $S_{l, m}(\lambda)$ are recorded at the two different outputs of the MMI, each one equipped with a FGC. Ideally both FGCs should have very similar performance. If this is not the case, a minor difference in the average power delivered from the FGCs changes Eq. (5) into:

$$
K_{l, m}^{ \pm}(\lambda)=\frac{\left(1 \pm \frac{\Delta}{2}\right) S_{l, m}(\lambda)}{\left(1 \mp \frac{\Delta}{2}\right) S_{l, 0}(\lambda)+\left(1 \pm \frac{\Delta}{2}\right) S_{l, 1}(\lambda)}
$$




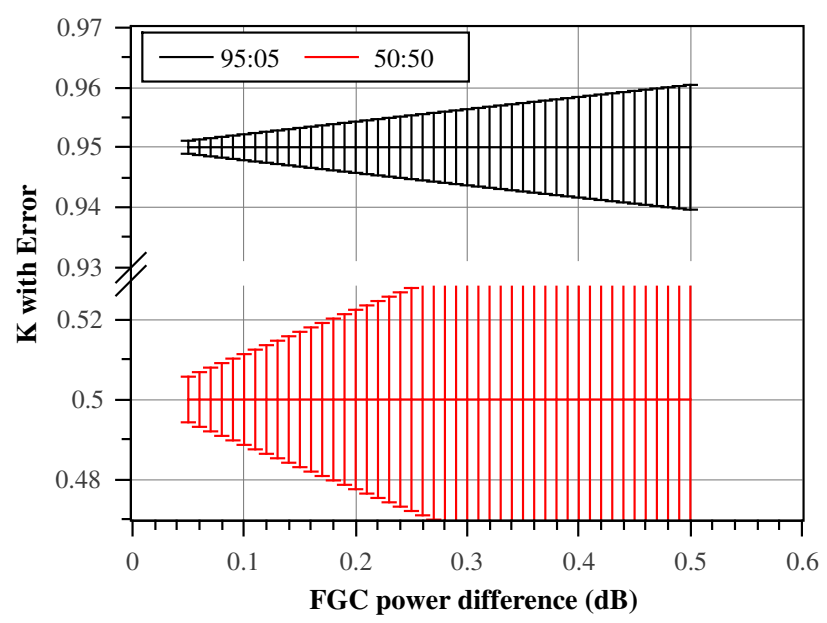

Fig. 5. Coupling ratio variation with average power difference delivered by the output FGCs, for nominal 50:50 and 95:05 coupling ratios.

where $\Delta$ represents difference between average power delivered by each FGC.

Hence, resorting to Fig. 5, the calculated coupling ratios, for a given difference in the performance of the FGCs, are more sensitive in the case of coupling ratios closer to 0.5. On the contrary, sensitivity to this issue is lower for more asymmetric couplers. This is clearly noticeable for MMI\#2 B1 in Fig. 6(b), MMI\#7 A3 in Fig. 7-(c), MMI\#8 A3 in Fig. 7-(d) as well.

Therefore the efficiency of the FGCs that may vary from one to other, not only between different dies, but inside the same die too [23], is the most likely cause of the cases out of the general trends. Similar conclusions can be inferred for MMIs \#5 to \#8 from Fig. 7.

\section{E. Excess loss}

An estimation for the excess loss, EL, is derived combining the MMI measured spectra, $S_{l, m}(\lambda)$, with the spectra of reference straight waveguides, $S_{s w}(\lambda)$ as.

$$
E L_{l}(\lambda)=10 \log _{10}\left[\frac{S_{s w}(\lambda)}{S_{l, 0}(\lambda)+S_{l, 1}(\lambda)}\right]
$$

with all the magnitudes in linear units. The spectra of four straight waveguides per die, spanning the same length than the MMIs with input/outputs, were measured. The maximum value at each wavelength was calculated to obtain a single trace $S_{s w}(\lambda)$ in each die for normalization. The average excess loss for all wavelength was then calculated. The result needed to be corrected by adding $0.4 \mathrm{~dB}$, meaning the deviations due to fiber alignments and differences in FGC performance are at least $0.4 \mathrm{~dB}$. Consequently, the EL values obtained are best case. It is likely the actual value to be $0.2-0.4 \mathrm{~dB}$ larger (i.e. despite considering the FGCs having equal performance, the fibers measuring the straight waveguides might be slightly misaligned). Under these conditions, the numerical values for the excess loss average for all wafers, dies and MMIs are calculated. Consequently, one cannot derive and absolutely accurate value for the EL from these measurements. Otherwise, resorting to full in depth statistical analysis of a larger number of samples would be required, and it is out of the scope of

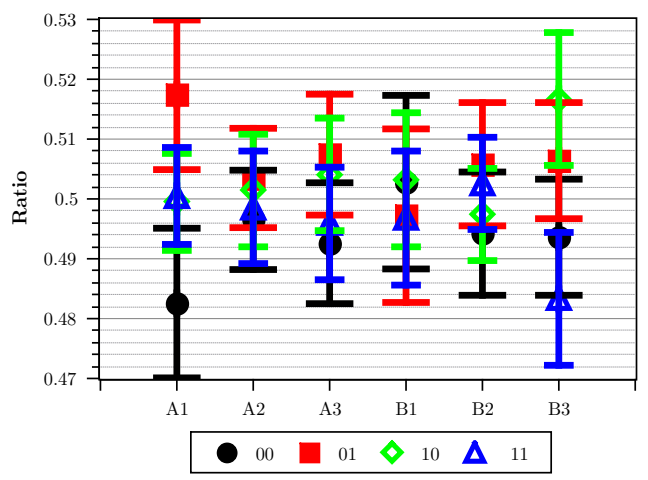

(a) MMI\#1

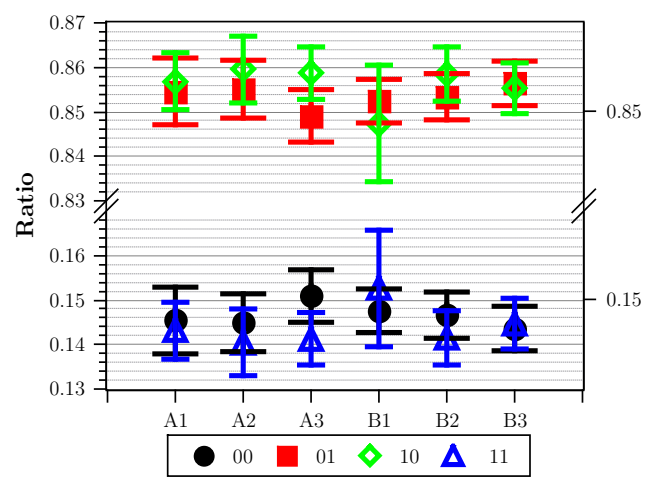

(b) MMI\#2

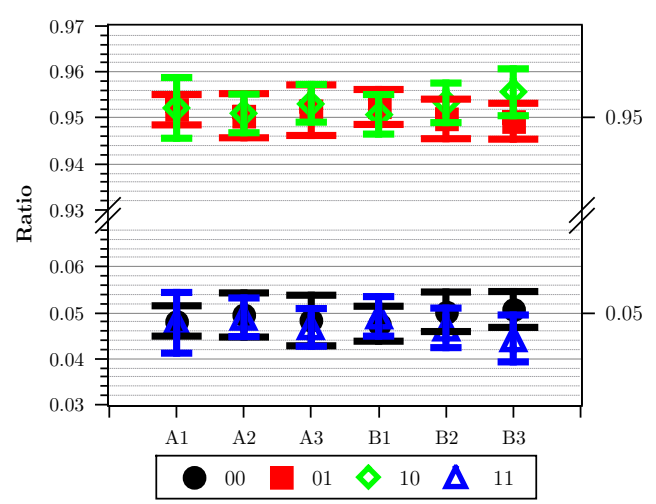

(c) MMI\#3

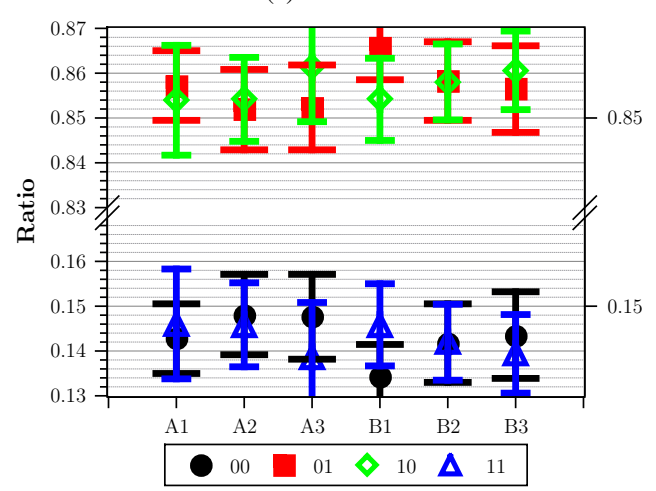

(d) MMI\#4

Fig. 6. MMIs \#1 to \#4, average and standard deviation in $\lambda \in$ $[1525,1575] \mathrm{nm}$ for the coupling ratios, for wafers A y B and dies 1, 2, 3 within each wafer. 


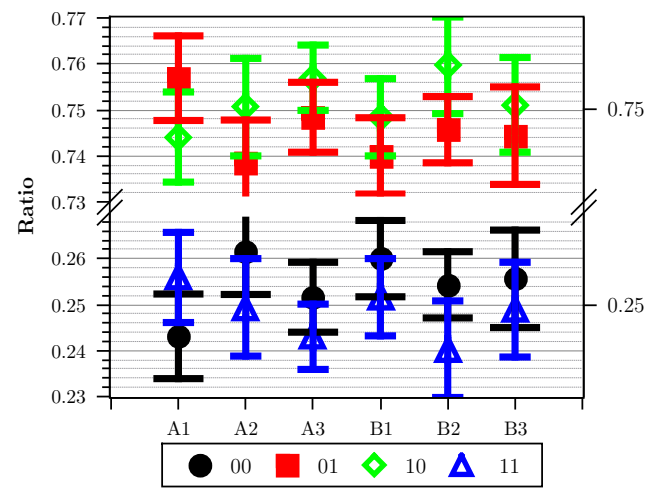

(a) MMI\#5

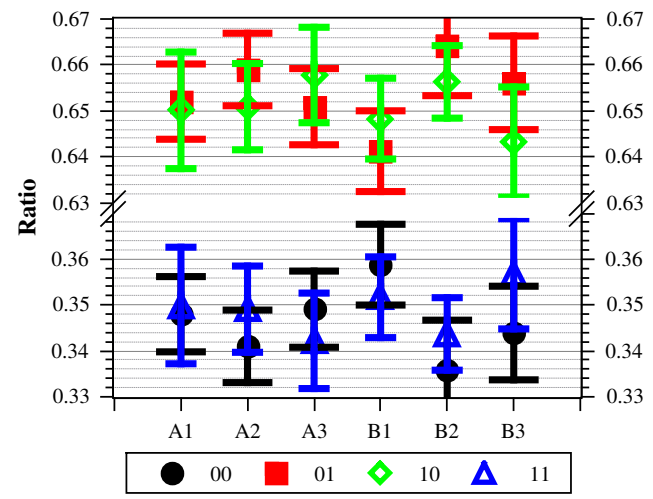

(b) MMI\#6

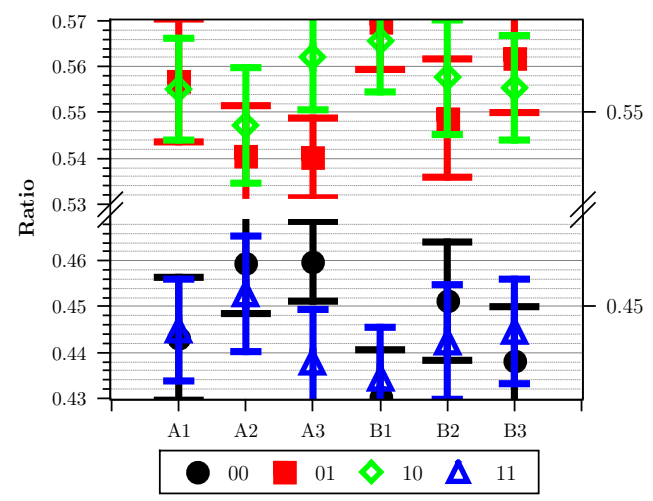

(c) MMI\#7

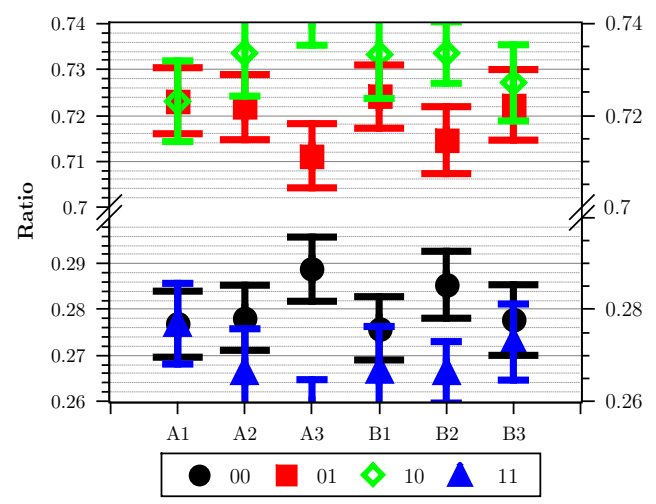

(d) MMI\#8

Fig. 7. MMIs \#5 to \#8, average and standard deviation in $\lambda \in$ $[1525,1575] \mathrm{nm}$ for the coupling ratios, for wafers A y B and dies 1, 2, 3 within each wafer.

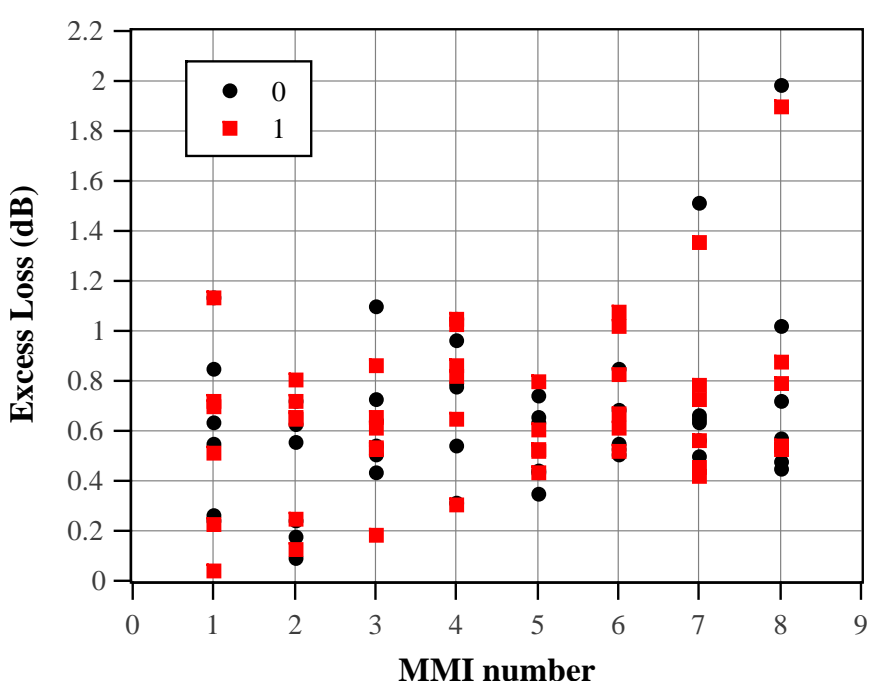

(a)

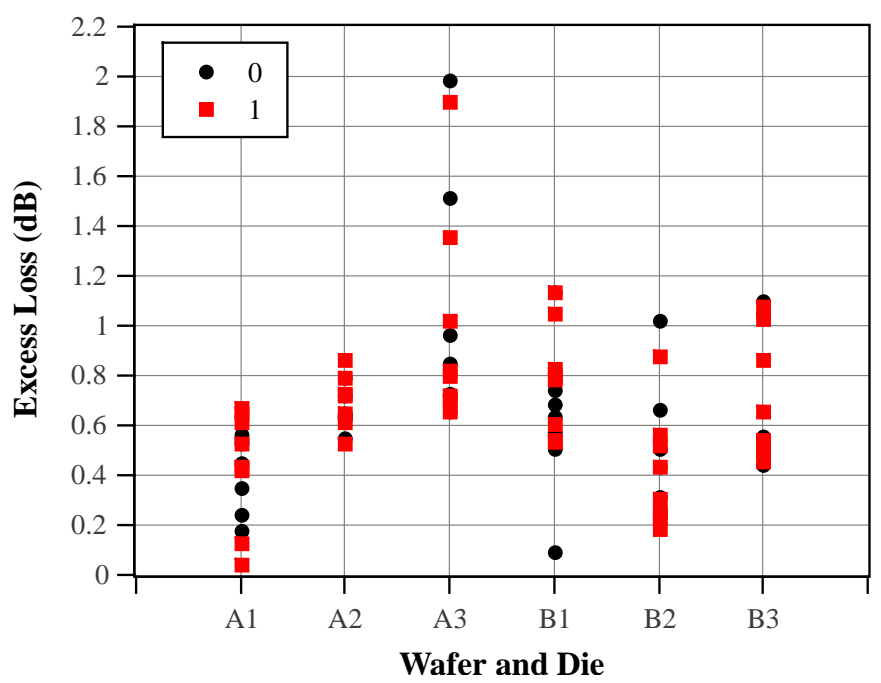

(b)

Fig. 8. Stacked values for the estimated excess loss for (a) each MMI, all dies and (b) each die, all MMIs.

this paper (see [24], [23] and [25] for reproducibility issues). From these calculations, the interval on which the EL lies can be estimated at the sight of Fig. 8. Panel (a) in the figure shows the estimated EL per MMI, i.e. all the dies for the each MMI. Panel (b) shows the estimated EL for all the MMIs in a die. Except MMI\#8 at die A3, Fig. 8-(a), which had excess loss close to $2 \mathrm{~dB}$, all the other devices/dies had losses in average around $0.6 \mathrm{~dB}$, since die A3 exhibited comparatively higher excess loss for all the MMIs, as can be inferred from Fig. 8-(b).

\section{F. Discussion}

The results in this paper constitute a library of designs for canonical and arbitrary coupling ration MMIs on Silicon. A comparison with previously reported works where parametric analysis is experimentally reported on Silicon follows.

In [12] the authors present a multiparametric analysis of 50:50 1x2 MMIs in SOI aiming to be ultra-compact. They report excess losses in the range of $0.1 \mathrm{~dB}$ to $0.6 \mathrm{~dB}$, which 
depends on the exposure doze during the fabrication process. The body widths range from $1.8 \mu \mathrm{m}$ to $3.2 \mu \mathrm{m}$, with body lengths below $10 \mu \mathrm{m}$, and using a deeply etched cross-section. Hence, the authors report optimization by means of Finite Difference in the Time Domain (FDTD), rather than BPM. The authors build an uneven 1x6 distribution network, by cascading $51 \times 2$ MMIs. Individual performance for the MMIs is reported in the C-band with a power imbalance of less than $0.2 \mathrm{~dB}$, defined as the ratio of the powers measured at the two outputs. From our results for the comparable 50:50 couplers, MMI\#1, the worst case imbalance is for die/wafer $\mathrm{A} 1$ and $\mathrm{B} 3$ with $10 \log _{10}(0.53 / 0.47)=0.52 \mathrm{~dB}$, but less than $10 \log _{10}(0.52 / 0.48)=0.34 \mathrm{~dB}$ for the rest of the dies.

In [13] the authors report on MZI-MMIs on a thick Silicon (4 $\mu \mathrm{m}$ core height) waveguides. The footprint of the devices is below $5 \times 300 \mu \mathrm{m}^{2}$. By means of a relative phase shift between the two arms of a MZI built with MMIs, the authors attain unconstrained coupling ratios. The coupling ratio is set through the length and widening/narrowing of one of the MZI arms. Variation analysis is provided for the phase shifter length. For a central wavelength of $1550 \mathrm{~nm}$, the following results are reported: power ratios reported are in the range of 45:55 to 100:0, similar to those in this paper; imbalance data is given for different phase shifter lengths, with variations of $\pm 1 \mathrm{~dB}$ between bar and cross outputs; and average insertion loss is $0.4 \mathrm{~dB}$ with $\mathrm{a} \pm 0.5 \mathrm{~dB}$ error interval. Uncertainties arising from measured spectral oscillations, attributed to the setup, are reported as well, similar to those described in this paper. Finally, spectral traces from $1450 \mathrm{~nm}$ up to $1650 \mathrm{~nm}$ are given, but wavelength dependent statistical information out of these traces, i.e. similar to the results in Fig. 6 and Fig. 7 is not directly inferred.

\section{CONCLUSION AND OUTLOOK}

In this paper the design, fabrication and measurement of MMIs with arbitrary coupling ratio in Silicon-on-Insulator technology has been reported. The design methodology consisted on a combination of theoretical first guess and numerical optimization, using the Beam Propagation Method. The devices were fabricated in two different wafers, where the waveguides had different etch depths. Very good match between the design and experimental results was obtained in terms of the coupling ratio for the devices. All the coupling ratios were attained within the design wavelength range of $1525-1575 \mathrm{~nm}$ with deviations as low as \pm 0.02 . Minor deviations were attributed to the difference in the performance of the focusing grating couplers. Except for one die, the estimated average excess loss for the MMIs is around $0.6 \mathrm{~dB}$. The results are comparable to other similar variation analyses reported in the literature.

The statistical and reproducibility information on this paper can be readily incorporated by others to device, circuit and network on-chip simulation and design tools, in order to asses on more complex photonic chip circuits based on these MMIs.

\section{ACKNOWLEDGMENT}

The authors acknowledge financial support by the Spanish CDTI NEOTEC start-up programme, the Spanish MICINN project TEC2010-21337, acronym ATOMIC, the Spanish MINECO project TEC2013-42332-P, acronym PIC4ESP, project FEDER UPVOV 10-3E-492 and project FEDER UPVOV 08-3E-008. B. Gargallo acknowledges financial support through FPI grant BES-2011-046100. J.S. Fandiño acknowledge financial support through FPU grant AP2010-1595.

\section{APPENDIX A \\ REFRACTIVE INDICES}

The following wavelength dependence for the refractive indices of the materials was included in the solver given by Sellmeier equation [26]:

$$
n^{2}(\lambda)=1+\sum_{i=0}^{N} \frac{B_{i} \lambda^{2}}{\lambda^{2}-C_{i}}
$$

For $\mathrm{Si}$ and $\lambda \in[1.36-11] \mu \mathrm{m}$ the coefficients are $\mathrm{B}_{i}=\{10.66842933, \quad 0.003043475, \quad 1.54133408\} \quad$ and $\mathrm{C}_{i}=\left\{0.3015116485^{2}, 1.13475115^{2}, 1104.0^{2}\right\} \mu \mathrm{m}^{2}$. For $\mathrm{SiO}_{2}$ and $\lambda \in[0.21-3.71] \mu \mathrm{m}$ the coefficients are $\mathrm{B}_{i}=\{0.6961663$, $0.4079426,0.1162414\}$ and $C_{i}=\left\{0.0684043^{2}, 0.1162414^{2}\right.$, $\left.9.896161^{2}\right\} \mu \mathrm{m}^{2}$.

\section{REFERENCES}

[1] P. Munoz, J. Domenech, I. Artundo, J. den Besten, and J. Capmany, "Evolution of fabless generic photonic integration," in Transparent Optical Networks (ICTON), 2013 15th International Conference on, June 2013, pp. 1-3.

[2] R. G. Hunsperger, Integrated optics: theory and technology. Springer, 2002.

[3] R. Ulrich and G. Ankele, "Self imaging in homogeneous planar optical waveguides," Applied Physics Letters, vol. 27, no. 6, 1975.

[4] E. Pennings, R. Deri, A. Scherer, R. Bhat, T. Hayes, N. Andreadakis, M. Smit, L. Soldano, and R. Hawkins, "Ultracompact, low-loss directional couplers on inp based on self-imaging by multimode interference," Applied physics letters, vol. 59, no. 16, pp. 1926-1928, 1991.

[5] L. B. Soldano and E. C. Pennings, "Optical multi-mode interference devices based on self-imaging: principles and applications," Lightwave Technology, Journal of, vol. 13, no. 4, pp. 615-627, 1995.

[6] M. Bachmann, P. Besse, and H. Melchior, "General self-imaging properties in $\mathrm{n} \times \mathrm{n}$ multimode interference couplers including phase relations," Applied Optics, vol. 33, no. 18, pp. 3905-3911, 1994.

[7] - "Overlapping-image multimode interference couplers with a reduced number of self-images for uniform and nonuniform power splitting," Applied optics, vol. 34, no. 30, pp. 6898-6910, 1995.

[8] P.-A. Besse, E. Gini, M. Bachmann, and H. Melchior, "New $2 \times 2$ and $1 \times 3$ multimode interference couplers with free selection of power splitting ratios," Lightwave Technology, Journal of, vol. 14, no. 10, pp. 2286-2293, Oct 1996.

[9] J. Leuthold and C. H. Joyner, "Multimode interference couplers with tunable power splitting ratios," J. Lightwave Technol., vol. 19, no. 5, p. 700, May 2001. [Online]. Available: http://jlt.osa.org/abstract.cfm?URI=jlt-19-5-700

[10] P.-A. Besse, M. Bachmann, H. Melchior, L. Soldano, and M. Smit, "Optical bandwidth and fabrication tolerances of multimode interference couplers," Lightwave Technology, Journal of, vol. 12, no. 6, pp. 10041009, Jun 1994.

[11] R. Halir, I. Molina-Fernandez, A. Ortega-Monux, J. Wanguemert-Perez, D.-X. Xu, P. Cheben, and S. Janz, "A design procedure for highperformance, rib-waveguide-based multimode interference couplers in silicon-on-insulator," Lightwave Technology, Journal of, vol. 26, no. 16, pp. 2928-2936, Aug 2008.

[12] H. Zhou, J. Song, C. Li, H. Zhang, and P. Lo, "A library of ultra-compact multimode interference optical couplers on SOI," Photonics Technology Letters, IEEE, vol. 25, no. 12, pp. 1149-1152, June 2013.

[13] M. Cherchi, S. Ylinen, M. Harjanne, M. Kapulainen, T. Vehmas, and T. Aalto, "Unconstrained splitting ratios in compact double-mmi couplers," Opt. Express, vol. 22, no. 8, pp. 9245-9253, Apr 2014. 
[14] "FieldDesigner and OptoDesigner software packages," http://www.phoenixbv.com/.

[15] M. T. Hill, X. Leijtens, G. D. Khoe, and M. K. Smit, "Optimizing imbalance and loss in $2 \times 23$-db multimode interference couplers via access waveguide width," Journal of lightwave technology, vol. 21, no. 10, p. 2305,2003

[16] D. Thomson, Y. Hu, G. Reed, and J.-M. Fedeli, "Low loss mmi couplers for high performance mzi modulators," Photonics Technology Letters, IEEE, vol. 22, no. 20, pp. 1485-1487, 2010

[17] W. Bogaerts, S. K. Selvaraja, P. Dumon, J. Brouckaert, K. De Vos, D. Van Thourhout, and R. Baets, "Silicon-on-insulator spectral filters fabricated with cmos technology," IEEE Journal of Selected Topics in Quantum Electronics, vol. 16, no. 1, pp. 33-44, 2010.

[18] P. Muñoz, R. García-Olcina, C. Habib, L. Chen, X. Leijtens, J. Doménech, M. Rius, J. Capmany, T. de Vries, M. Heck et al., "Sagnac loop reflector and arrayed waveguide grating-based multi-wavelength laser monolithically integrated on inp," IET optoelectronics, vol. 5, no. 5, pp. 207-210, 2011.

[19] J. Buus, "The effective index method and its application to semiconductor lasers," Quantum Electronics, IEEE Journal of, vol. 18, no. 7, pp. 1083-1089, 1982.

[20] Y. Fu, T. Ye, W. Tang, and T. Chu, "Efficient adiabatic silicon-on-insulator waveguide taper," Photon. Res., vol. 2, no. 3, pp. A41-A44, Jun 2014. [Online]. Available: http://www.opticsinfobase.org/prj/abstract.cfm?URI=prj-2-3-A41

[21] F. Van Laere, T. Claes, J. Schrauwen, S. Scheerlinck, W. Bogaerts, D. Taillaert, L. O'Faolain, D. Van Thourhout, and R. Baets, "Compact focusing grating couplers for silicon-on-insulator integrated circuits," Photonics Technology Letters, IEEE, vol. 19, no. 23, pp. 1919-1921, 2007.

[22] J. Domenech, "Apodized coupled resonator optical waveguides: Theory, design and characterization," Ph.D. dissertation, Universitat Politecnica de Valencia, http://hdl.handle.net/10251/32278, 2013.

[23] L. He, Y. Liu, C. Galland, A.-J. Lim, G.-Q. Lo, T. Baehr-Jones, and M. Hochberg, "A high-efficiency nonuniform grating coupler realized with 248-nm optical lithography," Photonics Technology Letters, IEEE, vol. 25, no. 14, pp. 1358-1361, 2013.

[24] P. Dumon, "Ultra-compact integrated optical filters in silicon-oninsulator by means of wafer-scale technology," Ph.D. dissertation, Universiteit Gent, 2007.

[25] W. Bogaerts, M. Fiers, and P. Dumon, "Design challenges in silicon photonics," Selected Topics in Quantum Electronics, IEEE Journal of, vol. 20, no. 4, pp. 1-8, July 2014.

[26] M. J. Weber, Handbook of optical materials. CRC press, 2002.

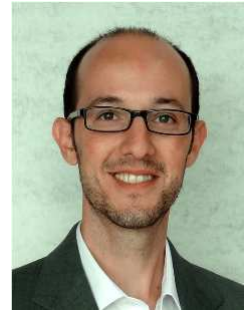

José David Doménech was born in Alfafar, Valencia (Spain). He received the B.Sc. degree in Telecommunications from the Escuela Politécnica Superior de Gandia (EPSG), Valencia, Spain, in 2006, the M.Sc. degree in Technologies, Systems and Networks of Communication from the Universidad Politcnica de Valencia (UPV), Valencia, Spain, in 2008 and the $\mathrm{Ph} . \mathrm{D}$. degree in photonics in 2013 at the Universitat Politècnica de València within the Optical and Quantum Communications Group. He has been involved in several national and international research projects. He has published over 10 papers in international refereed journals and over 20 conference contributions. J.D. Doménech received the Intel doctoral student honor programme award in 2012 in recognition for his work during his Ph.D. He is co-founder and CTO of the spin-off company VLC Photonics, devoted to the design of photonics integrated circuits in multiple integration technologies.

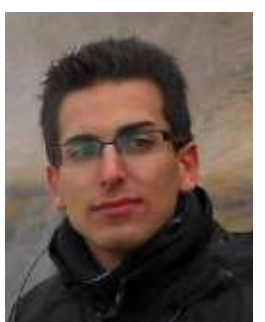

Javier S. Fandiño was born in Orihuela (Alicante), in 1987. He received the bachelor degree in Telecommunications engineering and the M.S. degree in Technology, Systems and Telecommunications Networks, both from the Universidad Politcnica de Valencia (Spain), in 2010 and 2011, respectively. In 2011, he joined the Optical and Quantum Communications group (GCOC), where he was first engaged in the experimental demonstration of an alloptical $2 \mathrm{R}$ regenerator for extended PON networks. At present, he is currently pursuing his $\mathrm{PhD}$ in telecommunications engineering in the GCOC, supported by the Spanish Ministry of Science and Innovation trough a FPU (Formacin de Personal Universitario) scholarship. His research topics include design and characterization of photonic integrated circuits (both in Silicon-On-Insulator and InGaAsP), and their potential applications in coherent optical communications and microwave photonics.

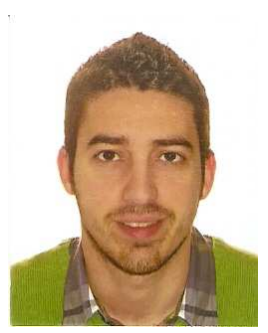

Bernardo Gargallo Bernardo Gargallo was born in Valencia, Spain, on August 29th, 1986. He received the M.Sc. in Telecommunication Engineering from the Universitat Politècnica de València (UPV) in 2011. He is currently pursuing the Ph.D. Degree at the Optical and Quantum Communications Group (OQCG), ITEAM Research Institute, UPV. His research has been focused on the design of integrated optical circuits to be applied in coherent communications, spectrometry and signal analysis.

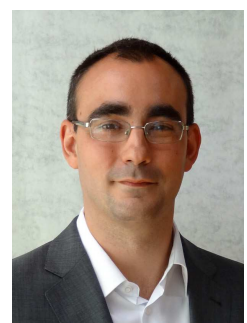

Pascual Muñoz was born in Valencia, Spain on February 7th, 1975. He received the Ingeniero de Telecomunicación degree from Universitat Politècnica de València (UPV) in 1998. In 1999 he served as 1st Lieutenant in the Spanish Airforce, while working as IT consultant for AIME Instituto Tecnológico. He received a Ph.D. degree in photonics from UPV in 2003. He is currently Associate Professor at the Departamento de Comunicaciones, and researcher at the Institute for Telecommunications and Multimedia Applications (ITEAM), both at UPV. Dr. Muñoz runs a consolidated research line, started in 2005, on prototyping Photonic Integrated Circuits (PICs) in a technology agnostic fashion, where PICs are designed in the best suited technology (Silicon-OnInsulator, Indium Phosphide, Silica on Silicon, Silicon Nitride amongst other) for each application. He has been involved in several European Commission funded projects, being coordinator for integration on InP within the NoE IST-EPIXNET. He has published over 25 papers in international refereed journals and over 40 conference contributions. $\mathrm{He}$ is a member of the Technical Programme Committees of the European Conference on Optical Communications (ECOC) and the European Conference on Integrated Optics (ECIO). Dr. Muñoz received the VPI Speed Up Photonics Award in 2002 for innovative Fourier optics AWG with multimode interference (MMI) couplers modeling, by Virtual Photonics Incorporated and IEEE Communications Magazine. He was also granted the IEEE/LEOS Graduate Student Fellowship Program in 2002. He received the extraordinary doctorate prize from UPV in 2006. From his research line, he co-founded the UPV spin-off company VLC Photonics in 2011, where the PIC design know-how, expertise and tools have been transferred, and he served as CEO from 2011 to 2013. Dr. Muñoz is a Senior Member of IEEE and Member of the OSA. 